\title{
A Dynamics Controller Design Method for Car-like Mobile Robot Formation Control
}

\author{
Baofang Wang , Chen Qian and Qingwei Chen \\ School of Automation, Nanjing University of Science and Technology, 210094 Nanjing, China
}

\begin{abstract}
A dynamics controller design method based on characteristic model is proposed for the formation control problem of car-like mobile robots. Only kinematics controller is not enough for some cases such as the environment is rugged, and the dynamics parameters of the robot are time-varying. Simulation results show that the proposed method can improve the responding speed of the mobile robots and maintain high formation accuracy. First, we obtain the kinematic error state equations according to the leader-follower method. A kinematics controller is designed and the stability is proved by Lyapunov theory. Then the characteristic model of the dynamics inner loop is established. A sliding mode controller is designed based on the second order discrete model, and the stability of the closed-loop system is analyzed. Finally, simulations are designed in MATLAB and Microsoft Robotics Developer Studio 4 (MRDS) to verify the effectiveness of the proposed method.
\end{abstract}

\section{Introduction}

Formation control problem of multi robots has become an interesting and challenging subject for researchers. In the wake of developments in sensors, servo motors and control technology, the performance of robot core components has been improved accordingly. Robots have been widely used in various situations.

In trajectory tracking and formation control problems for car-like mobile robots, numerous researchers have focused on the design of kinematics controllers. This is reasonable for many cases because of two factors: Firstly, most car-like robots have their own motor drive and control modules which contain the dynamics inner control loop capable of tracing the velocity command signal; Secondly, traditional robot workspaces are flat ground, and the mass and velocity of the robot are always low. In these situations, kinematics controllers are enough for robot trajectory tracking and formation control. Dynamics analysis is not necessary. However, in recent years, car-like robots have faced increasingly complicated situations due to the requirements of different tasks [1-3], such as geographical exploration, rescue after disaster and agriculture cultivation. Robots may have higher velocity and larger mass of load, and the workspaces are always rough terrain with obstacles. In these tasks, only kinematics controllers cannot satisfy the performance requirement. And researches on dynamics controllers become more and more necessary.
Characteristic model based adaptive controller design method is proposed in [4]. A second order discrete time-varying model is acquired by parameter identification. The characteristic model is established according to the input and output signals of the system without accurate mathematical system model. And the controller based on this model has good adaptive ability to the change of system parameters. So, when the dynamics model of the robot is unknown and the mass and velocity of the robot are time-varying, the characteristic model based dynamics controller design method may have some advantages.

Numerous researchers have focused on the kinematics controller design for car-like mobile robot formation control problem. In [5], a new vision based formation system was proposed. An adaptive kinematics controller and an adaptive camera controller were designed. The authors of [6] proposed a PD-type controller for car-like mobile robots to compensate the delay effect of data transmission, and the stability of the system was proved. Back-stepping method was used to design car-like robots kinematics controller in [7]. Besides kinematics controllers, some researchers have considered the dynamics controller design problem. A nonlinear control law was derived using Lyapunov analysis for formation control of car-like mobile robots using robot dynamics in [8]. The authors of [9] presented a sliding mode tracking control scheme using the simplified kinematics model of car-like robots.

In this paper, we propose a dynamics controller design method for car-like robots considering the time-varying dynamics parameters and the rough terrain workspace. 
According to the leader-follower method, the controller of outer kinematics loop is designed based on the error state equations. In the inner dynamics loop, a dynamics controller or compensation controller is proposed based on the characteristic model. The proposed method is tested using MRDS and MATLAB to verify the effectiveness of the proposed controller.

\section{Kinematics model and controller}

Using the typical triangle formation as an example, the leader-follower method and the model of the car-like mobile robot are shown in Figure 1. The rear wheels of the robot are fixed parallel to the car body and allowed to roll or spin but not slip. The front wheels can turn to the left or right, but the two wheels must be parallel or satisfy Ackermann steering geometry. For the convenience of explanation, we only consider the situation of parallel front wheels. All the corresponding kinematics parameters are also presented in Figure 1. $l$ is the wheelbase. $p=[x, y, \theta]^{\mathrm{T}}$ represents the state of the car-like mobile robot, where $(x, y)^{\mathrm{T}}$ is the position of the rear wheel centre and $\theta$ is the angle between the vehicle frame orientation and axis $\mathrm{X}$.

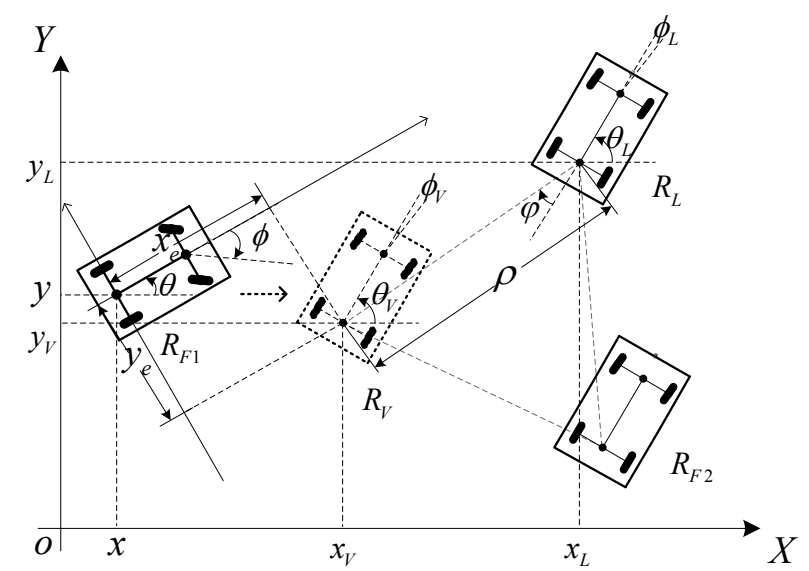

Figure 1. Leader-follower method.

$R_{\mathrm{L}}$ is the leader robot. $R_{\mathrm{F} 1}$ and $R_{\mathrm{F} 2}$ are the followers which need to keep triangle formation with the leader robot. Taking $R_{\mathrm{F} 1}$ as an example, $R_{\mathrm{V}}$ is the virtual robot which $R_{\mathrm{F} 1}$ should follow. If the position error $\mathbf{e}^{*}=\left[x_{\mathrm{V}}-x, y_{\mathrm{V}}-y, \theta_{\mathrm{V}}-\theta\right]^{\mathrm{T}}$ converge to zero, the formation is accomplished. It is the same with $R_{\mathrm{F} 2}$. The position of $R_{\mathrm{V}}$ is $\left[x_{\mathrm{V}}, y_{\mathrm{V}}, \theta_{\mathrm{V}}\right]^{\mathrm{T}}$ which can be acquired by the position of $R_{\mathrm{L}}$ and the formation parameters $[\rho, \varphi]^{\mathrm{T}}$ as follows,

$$
\left\{\begin{array}{l}
x_{\mathrm{V}}=x_{\mathrm{L}}-\rho \cos \left(\theta_{\mathrm{L}}+\varphi\right) \\
y_{\mathrm{V}}=y_{\mathrm{L}}-\rho \sin \left(\theta_{\mathrm{L}}+\varphi\right), \\
\theta_{\mathrm{V}}=\theta_{\mathrm{L}}
\end{array},\left\{\begin{array}{l}
v_{\mathrm{V}}=v_{\mathrm{L}} \\
\phi_{\mathrm{V}}=\phi_{\mathrm{L}}
\end{array} .\right.\right.
$$

The control input of the car-like robot is $[v, \phi]^{\mathrm{T}}$, where $v$ is the velocity of the rear wheels and $\phi$ is the steering angle of the front wheels. The kinematics model of the car-like mobile robot is as follows [8],

$$
\left\{\begin{array}{l}
\dot{x}=v \cos \theta \\
\dot{y}=v \sin \theta \\
\dot{\theta}=v \tan \phi / l
\end{array} .\right.
$$

Transform the coordinate from the global coordinate to the robot coordinate, the position error $\mathbf{e}^{{ }^{*}}$ is transformed into

$$
\mathbf{e}=\left[\begin{array}{l}
x_{\mathrm{e}} \\
y_{\mathrm{e}} \\
\theta_{\mathrm{e}}
\end{array}\right]=\mathbf{T e}^{*}, \quad \mathbf{T}=\left[\begin{array}{ccc}
\cos \theta & \sin \theta & 0 \\
-\sin \theta & \cos \theta & 0 \\
0 & 0 & 1
\end{array}\right] .
$$

Take the derivative of (3) and combine with the kinematics model (1), the error state functions can be obtained as

$$
\dot{\mathbf{e}}=\left[\begin{array}{c}
\dot{x}_{\mathrm{e}} \\
\dot{y}_{\mathrm{e}} \\
\dot{\theta}_{\mathrm{e}}
\end{array}\right]=\left[\begin{array}{l}
v_{\mathrm{v}} \cos \theta_{\mathrm{e}}+v y_{\mathrm{e}} \tan \phi / l-v \\
v_{\mathrm{v}} \sin \theta_{\mathrm{e}}-v x_{\mathrm{e}} \tan \phi / l \\
v_{\mathrm{v}} \tan \phi_{\mathrm{v}} / l-v \tan \phi / l
\end{array}\right] .
$$

The kinematics controller is designed as

$$
\left\{\begin{array}{l}
v=v_{\mathrm{v}} \cos \theta_{\mathrm{e}}+k_{1} x_{\mathrm{e}} \\
\phi=\arctan \left(\frac{v_{\mathrm{V}}}{v} \tan \phi_{\mathrm{v}}+\frac{k_{2} l v_{\mathrm{V}}}{\theta_{\mathrm{e}} v} y_{\mathrm{e}} \sin \theta_{\mathrm{e}}+\frac{k_{3} l}{v} \theta_{\mathrm{e}}\right),
\end{array}\right.
$$

where $k_{1}, k_{2}, k_{3}>0$. Consider the following Lyapunov function

$$
V=\frac{1}{2} x_{\mathrm{e}}^{2}+\frac{1}{2} y_{\mathrm{e}}^{2}+\frac{1}{2 k_{2}} \theta_{\mathrm{e}}^{2} .
$$

Combining (4), the derivative of (6) is given by

$$
\begin{aligned}
\dot{V}= & x_{\mathrm{e}} \dot{x}_{\mathrm{e}}+y_{\mathrm{e}} \dot{y}_{\mathrm{e}}+\frac{1}{k_{2}} \theta_{\mathrm{e}} \dot{\theta}_{\mathrm{e}} \\
= & x_{\mathrm{e}} v_{\mathrm{V}} \cos \theta_{\mathrm{e}}-x_{\mathrm{e}} v+y_{\mathrm{e}} v_{\mathrm{V}} \sin \theta_{\mathrm{e}} \\
& +\frac{1}{k_{2}} \theta_{\mathrm{e}}\left(v_{\mathrm{V}} \tan \phi_{\mathrm{V}} / l-v \tan \phi / l\right) \\
= & -x_{\mathrm{e}}\left(v-v_{\mathrm{v}} \cos \theta_{\mathrm{e}}\right)-\frac{\theta_{\mathrm{e}} v}{k_{2} l}(\tan \phi \\
& \left.-\frac{v_{\mathrm{V}}}{v} \tan \phi_{\mathrm{V}}-\frac{k_{2} l v_{\mathrm{V}}}{\theta_{\mathrm{e}} v} y_{\mathrm{e}} \sin \theta_{\mathrm{e}}\right)
\end{aligned}
$$

Taking the controller (5) into (7), we have

$$
\dot{V}=-k_{1} x_{\mathrm{e}}^{2}-\frac{k_{3}}{k_{2}} \theta_{\mathrm{e}}^{2} \leq 0 .
$$

Therefore, when the kinematics controller is selected as (5), the Lyapunov function $\dot{V} \leq 0$ and $\dot{V}=0$ only if $x_{\mathrm{e}}=0, \theta_{\mathrm{e}}=0$. So the kinematics outer loop is asymptotically stable.

\section{Dynamics model and controller}

As car-like mobile robots are widely used in different fields, there appear more and more situations when the dynamics parameters of the robot are time-varying, such as sprinklers, road sweeping robots and other transport 
robots with time-varying load. In these cases, the load of the robot is time-varying which influences the rotary inertia of the robot wheels and the whole mass of the robot. Therefore, an adaptive dynamics controller is designed to improve the accuracy of formation control.

In some cases, we should directly design the kinematics controller based on the characteristic model acquired by system identification. The system structure of dynamics controller design is shown in Figure 2.

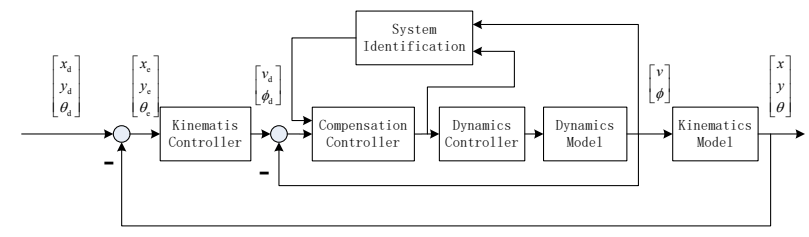

Figure 2. System structure of dynamics controller design.

When the robots have their own drive and control modules, we could not change their original dynamics controller. In this case, a dynamics compensation controller is designed to improve the control performance. The system structure of compensation controller design is shown in Figure 3.

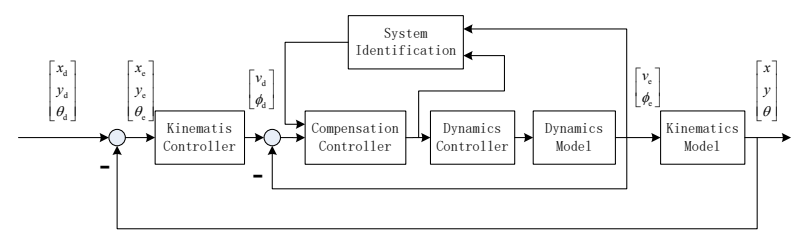

Figure 3. System structure of compensation controller design.

In Figure 2 and $3,\left[x_{\mathrm{d}}, y_{\mathrm{d}}, \theta_{\mathrm{d}}\right]^{\mathrm{T}}$ represents the expected position. $\left[x_{\mathrm{e}}, y_{\mathrm{e}}, \theta_{\mathrm{e}}\right]^{\mathrm{T}}$ is the position error. $\left[v_{\mathrm{d}}, \phi_{\mathrm{d}}\right]^{\mathrm{T}}$ is the expected input signal of kinematics outer control loop. $[v, \phi]^{\mathrm{T}}$ is the actual kinematics input signal, and $[x, y, \theta]^{\mathrm{T}}$ is the actual position of the robot.

Characteristic modeling method is based on the dynamic characteristics and control performance requirements of the plants. The characteristic model of the robot dynamics loop is acquired through system identification. It compresses the information of high-order model into several characteristic parameters which is different from the reduced-order model of a high-order system. Characteristic modeling method offers a way to design a feasible low-order controller for various complicated plants, and has been successfully applied to many situations. When the system satisfies the following conditions, it can be expressed as a second-order discrete time-varying system.

Consider a nonlinear system

$$
\dot{x}(t)=f\left(x, \dot{x}, \cdots, x^{(n)}, u, \dot{u}, \cdots, u^{(m)}\right) .
$$

Define

$$
\begin{aligned}
& x=x_{1}, \dot{x}=x_{2}, \cdots, x^{(n)}=x_{n+1} \\
& u=u_{1}, \dot{u}=u_{2}, \cdots, u^{(m)}=u_{m+1}
\end{aligned}
$$

Then (9) can be written as

$$
\dot{x}_{1}(t)=f\left(x_{1}, \cdots, x_{n+1}, u_{1}, \cdots, u_{m+1}\right) .
$$

Assuming the system satisfies the following conditions:

(a) Single input single output;

(b) The order of the control input $\mathrm{u}$ is 1 ;

(c) $f(\square)=0$ when all the variables $x_{i}=u_{i}=0$;

(d) $f(\square)$ is continuous and differentiable to all the variables $x_{i}$ and $u_{i}$, and all partial derivatives are bounded;

(e) $|f(x(t+T), u(t+T))|-|f(x(t), u(t))|<M T$, where $T$ is the sampling period and $\mathrm{M}$ is a positive constant.

Lemma 1 [4]. If a system can be described by (11) and satisfy the assumptions (a)-(e), then the characteristic model of the system can be established in the form of a second-order difference equation as

$$
\begin{aligned}
x(k+1)= & f_{1}(k) x(k)+f_{2}(k) x(k-1) \\
& +g_{0}(k) u(k)+g_{1}(k) u(k-1)
\end{aligned} .
$$

In the dynamic process, under the same input, the error between characteristic model output and the practical plant output is maintained within a permitted small range. In the steady state, the two outputs are equal. The parameters $f_{1}(k), f_{2}(k), g_{0}(k), g_{1}(k)$ are slow time-varying. If the system is a minimum phase system, (12) can be simplified as

$$
x(k+1)=f_{1}(k) x(k)+f_{2}(k) x(k-1)+g_{0}(k) u(k) .
$$

According to the Newton's second law, the simplified car-like robot dynamics model can be easily obtained. The assumption (b) and (c) are satisfied. The velocity of the rear wheels and the steering angle of the front wheels are independent from each other. Therefore, the dynamics model can be considered as two systems with single input and single output, which satisfies the assumption (a). In practical situations, the velocity and angular velocity of the car-like mobile robot cannot change suddenly because of inertia. In most cases, all the parameters are continuous and smooth which satisfies (d). And assumption (e) is automatically satisfied.

We use the rear wheel velocity control system as an example to design a dynamics controller, and the steering angle control system of front wheels are the same. The error system is described as

$$
e(k+1)=f_{1}(k) e(k)+f_{2}(k) e(k-1)+g_{0}(k) u(k) .
$$

The parameters are estimated using least squares algorithm, and we have

$$
\begin{aligned}
e(k+1)= & \hat{f}_{1}(k) e(k)+\hat{f}_{2}(k) e(k-1), \\
& +\hat{g}_{0}(k) u(k)+\Delta(k)
\end{aligned}
$$

where $e(k)=x(k)-x_{r}(k) . x(k)$ is the actual velocity output, and $x_{r}(k)$ is the expected velocity. $\Delta(k)$ represents the error of parameter estimation, uncertainty and disturbance. 
The controller of system (14) is written as the following form

$$
u(k)=u_{\text {eq }}(k)+u_{\mathrm{s}}(k) .
$$

The adaptive control law is

$$
u_{\mathrm{eq}}(k)=-\frac{1}{\hat{g}_{0}(k)}\left[\hat{f}_{1}(k) e(k)+\hat{f}_{2}(k) e(k-1)\right] .
$$

And the sliding mode control law is

$$
u_{\mathrm{s}}(k)=\frac{1}{\hat{g}_{0}(k)}\{(1-q T) e(k)-\varepsilon T \operatorname{sgn}[e(k)]\} .
$$

Assuming $|\Delta(k)|<f_{\mathrm{m}}$, the parameters should satisfy the following ranges:

$$
\left\{\begin{array}{l}
T<\frac{2\left|e_{0}\right|}{2 \varepsilon+q\left|e_{0}\right|} \\
q>0 \\
\varepsilon>\frac{f_{\mathrm{m}}}{T}
\end{array} .\right.
$$

In (19), sgn( $\square$ ) represents the sign function. $e_{0}$ is the minimum error. $T$ is the sampling period, and $\varepsilon, q$ are positive constants.

Theorem 1.Suppose the system can be described as (14), and the control law (16) is used with all parameters satisfying (19), then the system is bounded stable.

Proof. Taking (16) into (15), we have

$$
e(k+1)=(1-q T) e(k)-\varepsilon T \operatorname{sgn}[e(k)]+\Delta(k) .
$$

Considering $[e(k+1)-e(k)] \operatorname{sgn}[e(k)]$, we can obtain

$$
\begin{aligned}
& {[e(k+1)-e(k)] \operatorname{sgn}[e(k)]} \\
& =-q T|e(k)|-\varepsilon T+\Delta(k) \operatorname{sgn}[e(k)] \\
& <-\varepsilon T+\Delta(k) \operatorname{sgn}[e(k)] \\
& <-\varepsilon T+f_{m}
\end{aligned}
$$

From the ranges of parameters (19), we know $\varepsilon>\frac{f_{\mathrm{m}}}{T}$. So $[e(k+1)-e(k)] \operatorname{sgn}[e(k)]<0$.

Then considering $[e(k+1)+e(k)] \operatorname{sgn}[e(k)]$, we have

$$
\begin{aligned}
& {[e(k+1)+e(k)] \operatorname{sgn}[e(k)]} \\
& =(2-q T)|e(k)|-\varepsilon T+\Delta(k) \operatorname{sgn}[e(k)] \\
& =(2-q T)|e(k)|-2 \varepsilon T+\varepsilon T+\Delta(k) \operatorname{sgn}[e(k)] . \\
& >(2-q T)|e(k)|-2 \varepsilon T+f_{\mathrm{m}}+\Delta(k) \operatorname{sgn}[e(k)] \\
& >(2-q T)|e(k)|-2 \varepsilon T
\end{aligned}
$$

From (19), $\quad T<\frac{2\left|e_{0}\right|}{2 \varepsilon+q\left|e_{0}\right|} \quad$ can be written as $2-q T>\frac{4 \varepsilon}{2 \varepsilon+q\left|e_{0}\right|}>0$, and $(2-q T)\left|e_{0}\right|-2 \varepsilon T>0$. Therefore,

$$
\begin{aligned}
& {[e(k+1)+e(k)] \operatorname{sgn}[e(k)]} \\
& >(2-q T)|e(k)|-2 \varepsilon T \\
& >(2-q T)\left|e_{0}\right|-2 \varepsilon T \\
& >0
\end{aligned}
$$

As a result, under the control law (16), the system satisfies

$$
\left\{\begin{array}{l}
{[e(k+1)-e(k)] \operatorname{sgn}[e(k)]<0} \\
{[e(k+1)+e(k)] \operatorname{sgn}[e(k)]>0}
\end{array} .\right.
$$

And the stability condition of discrete sliding mode control is satisfied [10].

\section{Simulation results}

Simulation1:

Two car-like mobile robots are expected to keep a certain formation and change to another formation at $t=60 \mathrm{~s}$. The mass of load is varying all the time during the simulation.

In the simulation, the wheelbase of the robot is $l=1.9 \mathrm{~m}$. The leader robot $R_{1}$ moves along a circle trajectory, and the following robot $R_{2}$ is controlled using the proposed control method to keep a certain formation with the leader. The initial formation parameters are $\rho=5 \mathrm{~m}$ and $\varphi=0^{\circ}$. The initial position error is $\left(x_{\mathrm{e}}, y_{\mathrm{e}}, \theta_{\mathrm{e}}\right)^{\mathrm{T}}=(5,5,0)^{\mathrm{T}}$. The initial load mass of $R_{2}$ is $m_{0}=2000 \mathrm{~kg}$ and decreases by $5 \mathrm{~kg}$ each second. When $t=60 \mathrm{~s}$, the formation parameters are changed to $\rho=5 \mathrm{~m}$ and $\varphi=-90^{\circ}$. The formation, position error and angle error are shown in Figure 4.

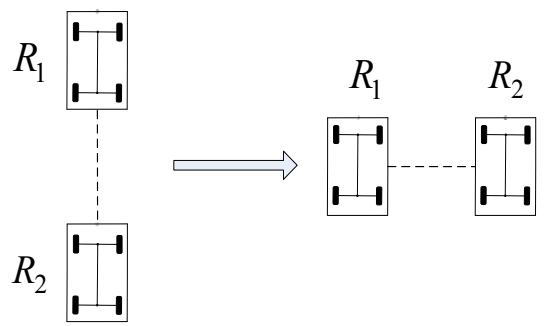

(a) Formation of car-like mobile robots

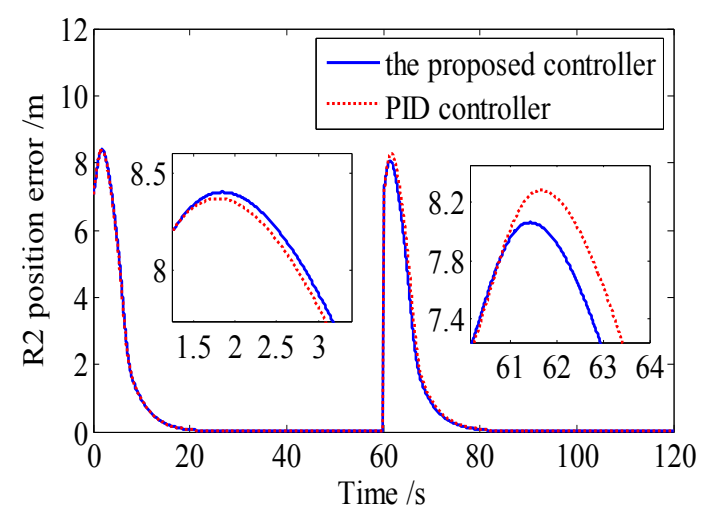

(b) Position error of $R_{2}$ 


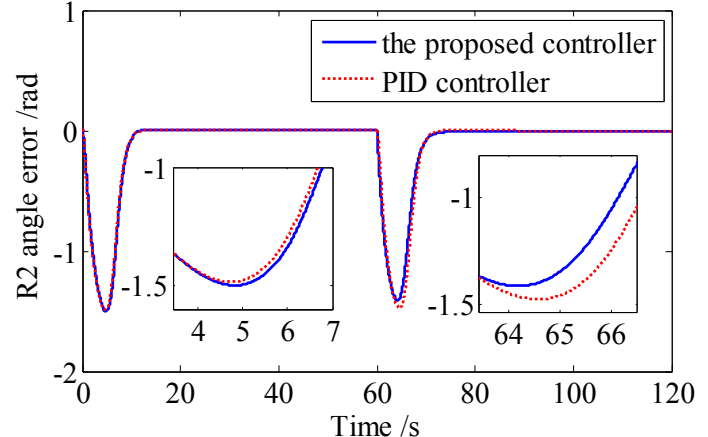

(c) Angel error of $R_{2}$

Figure 4. Simulation results using time-varying dynamics parameters.

From (b) and (c) of Figure 4, we can see that when $t<60$ s, the two controllers both can keep the expected formation with high accuracy. However, when $t>60 \mathrm{~s}$, PID controller cannot adjust to the change of dynamics parameters. Its speed of convergence becomes lower. And the proposed controller based on the characteristic model has good adaptive ability which makes it faster converge to the expected formation position. The proposed method has better control performance when the dynamics parameters are time-varying.

Simulation 2:

In practical, the work environments of car-like mobile robots are always rough terrain workspaces which have higher requirements for robot dynamics controller. In this simulation, a compensation dynamics controller is designed for robot formation control. Virtual 3D simulation environments are built in MRDS4 which provides direct debugger interface to MATLAB and has a physics engine PhysX making the simulation more realistic. The simulation environment are shown in Figure 5.

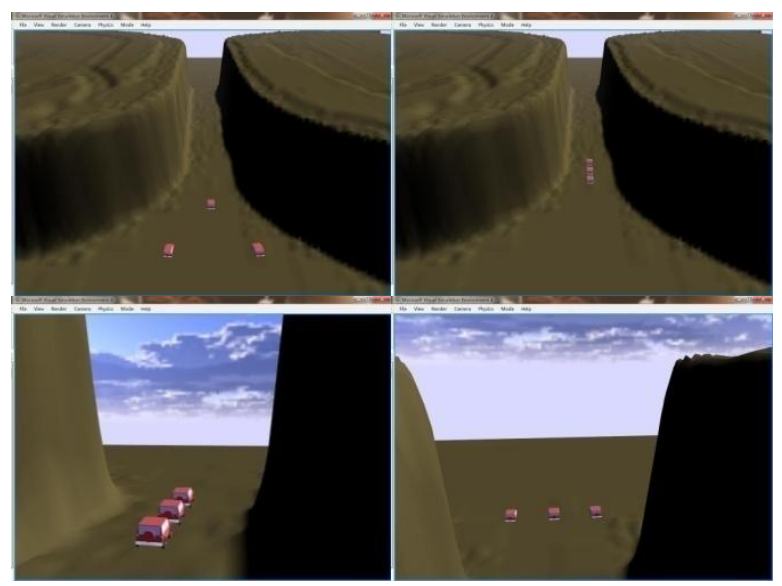

Figure 5. Simulation environment.

In this simulation, the wheelbase of the robot is $l=1.9 \mathrm{~m}$. Three robots $R_{1}, R_{2}, R_{3}$ go through a narrow canyon under expected formations. The initial formation parameters of $R_{2}, R_{3}$ are $\rho=7 \mathrm{~m}, \varphi_{2}=30^{\circ}, \varphi_{3}=-30^{\circ}$;
When the robots are in the canyon area, the formation parameters are $\rho_{2}=5 \mathrm{~m}, \rho_{3}=10 \mathrm{~m} \varphi=0^{\circ}$; After the canyon area, the formation parameters are changed to $\rho=7 \mathrm{~m}, \varphi_{2}=90^{\circ}, \varphi_{3}=-90^{\circ}$. The formation, trajectories, position errors and angle errors are shown in Figure 6.

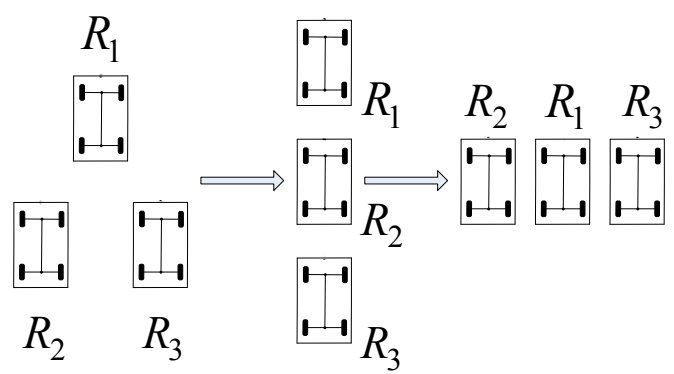

(a) Formation of car-like mobile robots

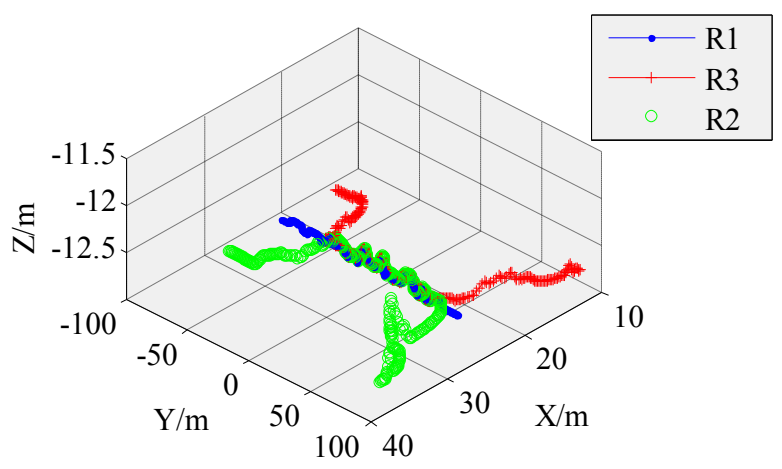

(b) Trajectories of robots

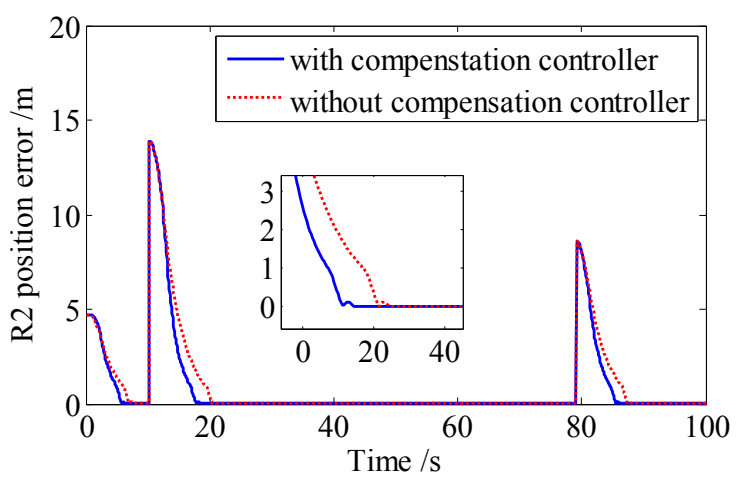

(c) Position error of $R_{2}$

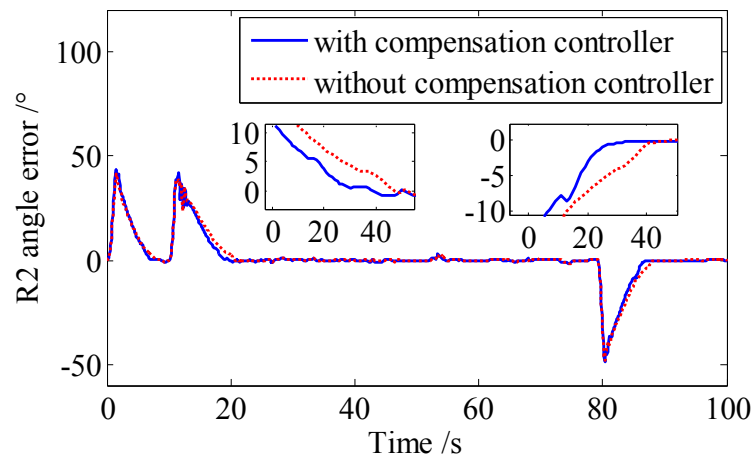

(d) Angle error of $R_{2}$ 


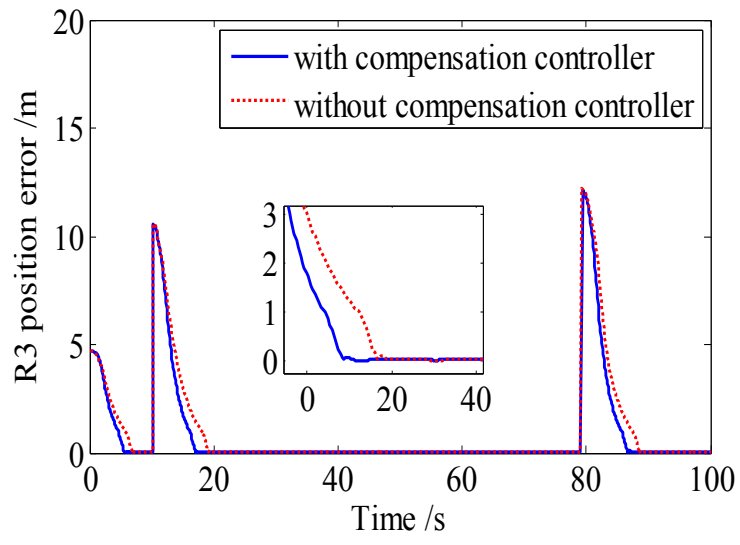

(e) Position error of $R_{3}$

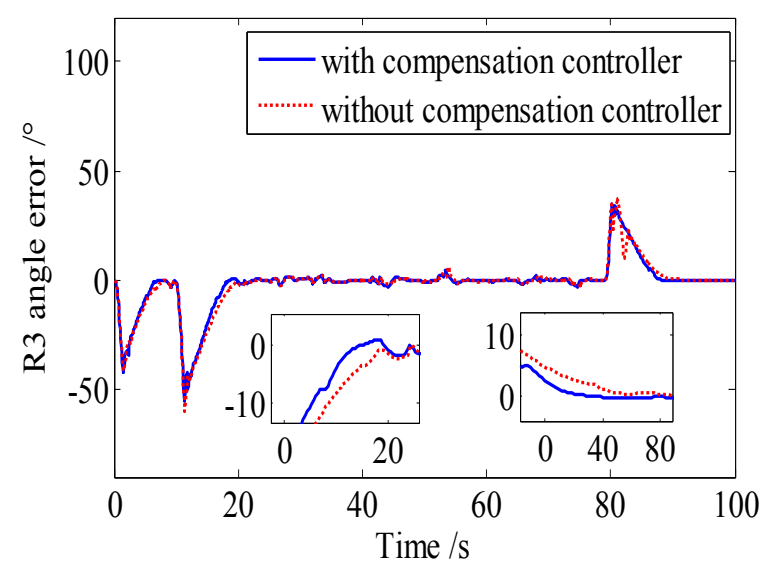

(f) Angle error of $R_{3}$

Figure 5. Simulation results in rough terrain environment.

From the simulation results, we know that rough terrain environment affects the accuracy of formation control. The position and angle errors cannot converge very fast when only the original dynamics controller is used. If we add the compensation controller based on characteristic model into the dynamics loop, the robots respond much faster making the convergence time shorter than without compensation.

\section{Conclusions}

In this paper, a dynamics controller design method based on characteristic model is proposed for the formation control problem of car-like mobile robots. First, we obtain the kinematic error state equations according to the leader-follower method. A kinematics controller is designed and the stability is proved by Lyapunov theory. Then the characteristic model of the dynamics inner loop is established. A sliding mode controller is designed based on the second order discrete model, and the stability of the closed-loop system is analyzed. Finally, two simulations are designed to verify the effectiveness of the proposed method. The proposed method can decrease the response time of robots and improve the control performance when the dynamics parameters are time-varying and the work environment is rough terrain.

\section{Acknowledgment}

This work was supported by National Natural Science Foundation of China under Grant No. 61333008, $61673214,61773211,61673217$ and 61673219.

\section{References}

1. C.R. Weisbin, G. Rodriguez, IEEE Rob. Autom Mag. 7, 25 (2002)

2. G.K. Dey, R. Hossen, M.S. Noor, K.T. Ahmmed, International Conference on Informatics, Electronics \& Vision, Dhaka, Bangladesh (IEEE, Dhaka Bangladesh, 2013)

3. I.A. Hameed, J. Intell. Robot. Syst. 74, 965 (2014)

4. H. Wu, J. Hu, Y. Xie, Characteristic model-based intelligent adaptive control (China Science and Technology Press, Beijing, 2009)

5. X. Chen, Y. Jia, IET Control Theory Appl. 9, 1302 (2015)

6. Z. Xu, M. Schroter, N. Dan, L. Ma, K. Schilling, Proceedings of the $31^{\text {st }}$ Chinese Control Conference (IEEE, Hefei China, 2012)

7. R. Zhang, S. Li, Q. Chen, Robot, 35, 651 (2013)

8. Z. Cao, Y. Zhao, Y. Fu, Acta Electron Sin, 40, 632 (2012)

9. S. Ramaswamy, S. Balakrishnan, American Control Conference (IEEE, Seattle America, 2008)

10. W. Gao, Y. Wang, A. Homaifa, IEEE Trans. Ind. Electron. 42, 117 (1995) 